\title{
Polynomial approximation on a compact subset of the real line
}

\author{
Vladimir Andrievskii
}

\begin{abstract}
We prove an analogue of the classical Bernstein theorem concerning the rate of polynomial approximation of piecewise analytic functions on a compact subset of the real line.
\end{abstract}

Keywords: Polynomial approximation, Green's function, Piecewise analytic function.

$M S C: 30 \mathrm{C} 10,30 \mathrm{E} 10$.

\section{Introduction and the main result}

Let $E \subset \mathbb{R}$ be a compact subset of the real line $\mathbb{R}$ and let $\mathbb{P}_{n}$ be the set of all (real) polynomials of degree at most $n \in \mathbb{N}:=\{1,2, \ldots\}$. Also, let for $x_{0} \in E$ and $\alpha>0$,

$$
\mathcal{E}_{n}\left(\left|x-x_{0}\right|^{\alpha}, E\right):=\inf _{p \in \mathbb{P}_{n}} \sup _{x \in E}|| x-\left.x_{0}\right|^{\alpha}-p(x) \mid .
$$

The starting point of our analysis is the classical Bernstein theory $[6,7,8]$. According to this theory, for any $x_{0} \in(-1,1)$ and $\alpha>0$, where $\alpha$ is not an even integer, there exists a finite nonzero limit

$$
\sigma_{\alpha}:=\lim _{n \rightarrow \infty} n^{\alpha} \mathcal{E}_{n}\left(\left|x-x_{0}\right|^{\alpha},[-1,1]\right) .
$$

The question as to what happens to the best polynomial approximations for a general set $E \subset \mathbb{R}$ is investigated in monographs [15] and [14, Chapter 10] where the reader can also find a comprehensive survey of this subject.

Now, we consider $E$ to be a set in the complex plane $\mathbb{C}$ and use the notions of potential theory in the plane (see $[12,13]$ for details). Let $E$ be non-polar, i.e., be of positive (logarithmic) capacity $\operatorname{cap}(E)>0$ and let $g_{\overline{\mathbb{C}} \backslash E}(z)=g_{\overline{\mathbb{C}} \backslash E}(z, \infty), z \in$ $\overline{\mathbb{C}} \backslash E$ be the Green function of $\overline{\mathbb{C}} \backslash E$ with pole at infinity, where $\overline{\mathbb{C}}:=\mathbb{C} \cup\{\infty\}$ is the extended complex plane.

Our main objective is to prove the following result. 
Theorem 1 Let $x_{0} \in E$. If for some $\alpha>0$,

$$
\limsup _{n \rightarrow \infty} n^{\alpha} \mathcal{E}_{n}\left(\left|x-x_{0}\right|^{\alpha}, E\right)>0
$$

then

$$
\sup _{z \in \mathbb{C} \backslash E} \frac{g_{\overline{\mathbb{C}} \backslash E}(z)}{\left|z-x_{0}\right|}<\infty
$$

Comparing Theorem 1 with [4, Corollary 1] we obtain the following result.

Theorem 2 Let $x_{0} \in E$. Then for any $\alpha>0$, which is not even integer,

$$
\liminf _{n \rightarrow \infty} n^{\alpha} \mathcal{E}_{n}\left(\left|x-x_{0}\right|^{\alpha}, E\right)>0
$$

if and only if (1.2) holds.

For the geometry of $E$ satisfying (1.2), we refer the reader to $[9,14,10,3]$ and the many references therein.

\section{Auxiliary results}

In this section we assume that

$$
E=\bigcup_{j=1}^{m}\left[a_{j}, b_{j}\right], \quad x_{0} \in \bigcup_{j=1}^{m}\left(a_{j}, b_{j}\right)=: \operatorname{Int}(E),
$$

where $-1=a_{1}<b_{1}<a_{2}<\ldots<b_{m-1}<a_{m}<b_{m}=1, m>1$.

It is known (for example, see [16, pp. 224-226], [5, pp. 409-412] or [2]) that there exists a conformal mapping $w=F(z)=F_{E}(z)$ of the upper half-plane $\mathbb{H}:=\{z: \Im z>0\}$ onto the domain

$$
G=G_{E}=\{z: 0<\Re z<\pi, \Im z>0\} \backslash \cup_{j=1}^{m-1}\left[u_{j}, u_{j}+i v_{j}\right],
$$

where $0=: u_{0}<u_{1}<u_{2}<\ldots<u_{m-1}<u_{m}:=\pi$ and $v_{j}>0, j=1, \ldots, m-$ 1, which can be extended continuously to $\overline{\mathbb{H}}$ satisfying the following boundary correspondence

$$
\begin{gathered}
F(\infty)=\infty, F((-\infty,-1])=\{z: \Re z=0, \Im z \geq 0\}, \\
F([1, \infty))=\{z: \Re z=\pi, \Im z \geq 0\}, \\
F\left(\left[a_{j}, b_{j}\right]\right)=\left[u_{j-1}, u_{j}\right], \quad j=1, \ldots, m, \\
F\left(\left[b_{j}, a_{j+1}\right]\right)=\left[u_{j}, u_{j}+i v_{j}\right], \quad j=1, \ldots, m-1 .
\end{gathered}
$$

Moreover,

$$
g_{\overline{\mathbb{C}} \backslash E}(z)=\Im(F(z)), \quad z \in \overline{\mathbb{H}}
$$




$$
\pi \mu_{E}([a, b])=|F([a, b] \cap E)|, \quad[a, b] \subset[-1,1],
$$

where $\mu_{E}$ is the equilibrium measure for $E$ and $|S|$ means the linear Lebesgue measure (length) of $S \subset \mathbb{C}$.

Since, by the reflection principle, $F$ can be extended analytically in a neighborhood of $x_{0}$, we can consider function

$$
h\left(x_{0}\right)=h_{E}\left(x_{0}\right):=F^{\prime}\left(x_{0}\right)=\pi \omega_{E}\left(x_{0}\right),
$$

where $\omega_{E}\left(x_{0}\right)$ is the density of $\mu_{E}$ at $x_{0}$.

Our interest in this function lies in the fact that by the Vasiliev - Totik theorem (see [15, p. 163, Corollary 11] and [14, Theorem 10.5]) for $\alpha>0$,

$$
\lim _{n \rightarrow \infty} n^{\alpha} \mathcal{E}_{n}\left(\left|x-x_{0}\right|^{\alpha}, E\right)=h\left(x_{0}\right)^{-\alpha} \sigma_{\alpha} .
$$

We set a sufficiently large constant $c>2$ such that

$$
h\left(x_{0}\right) \leq c \quad \text { and } \quad \operatorname{cap}(E)>\frac{1}{c} .
$$

In what follows, we denote by $c_{1}, c_{2}, \ldots$ positive constants which depend only on c.

By virtue of $[2$, p. 39, (1.8)], for $j=1, \ldots, m-1$,

$$
\begin{aligned}
v_{j} & \leq \log \left(\frac{1+(1-2 \operatorname{cap}(E))^{1 / 2}}{1-(1-2 \operatorname{cap}(E))^{1 / 2}}\right) \\
& \leq \log \left(\frac{\sqrt{c}+\sqrt{c-2}}{\sqrt{c}-\sqrt{c-2}}\right)<\log (2 c) .
\end{aligned}
$$

For the fixed point $z_{0}:=x_{0}+2 c e^{4 \pi} i \in \mathbb{H}, w_{0}:=F\left(z_{0}\right)$, and $\mathbb{D}:=\{z:|z|<1\}$, we have

$$
\begin{aligned}
\Im\left(w_{0}\right) & =g_{\overline{\mathbb{C}} \backslash E}\left(z_{0}\right) \geq g_{\overline{\mathbb{C}} \backslash \overline{\mathbb{D}}}\left(z_{0}\right)=\log \left|z_{0}\right| \geq \log \left|z_{0}-x_{0}\right| \\
& =4 \pi+\log (2 c)=: c_{1}>v_{j}+4 \pi, \quad j=1, \ldots, m-1 .
\end{aligned}
$$

Meanwhile, by virtue of [13, p. 53] and (2.2),

$$
\begin{aligned}
\Im\left(w_{0}\right) & =g_{\overline{\mathbb{C}} \backslash E}\left(z_{0}\right)=\int \log \left|z_{0}-x\right| d \mu_{E}(x)-\log \operatorname{cap}(E) \\
& \leq \log \left(\left|z_{0}-x_{0}\right|+2\right)+\log c<\log \left(4 c^{2}\right)+4 \pi<2 c_{1} .
\end{aligned}
$$

We use the notion of the module of a family of curves. We refer to [1, Chapter 4], [11, Chapter 9] or [5, pp. 341-360] for the definition and basic properties of the module (such as conformal invariance, comparison principle, composition 
laws, etc.) We use this properties without further citation. The curves under consideration are crosscuts (see [11]) either of $\mathbb{H}$ or of $G$.

For $z_{1}, z_{2} \in \mathbb{H}$ denote by $\Gamma\left(x_{0}, z_{1} ; z_{2}, \infty ; \mathbb{H}\right)$ the family of all crosscuts of $\mathbb{H}$ that separate points $x_{0}$ and $z_{1}$ from $z_{2}$ and $\infty$ in $\mathbb{H}$. Note that if $\left|z_{1}-x_{0}\right|<\left|z_{2}-x_{0}\right|$, then for the module of $\Gamma:=\Gamma\left(x_{0}, z_{1} ; z_{2}, \infty ; \mathbb{H}\right)$ we have

$$
0 \leq m(\Gamma)-\frac{1}{\pi} \log \left|\frac{z_{2}-x_{0}}{z_{1}-x_{0}}\right| \leq 2 .
$$

Indeed, let $\Gamma_{0} \subset \Gamma$ denote the family of all crosscuts of $A:=\left\{z \in \mathbb{H}:\left|z_{1}-x_{0}\right|<\right.$ $\left.\left|z-x_{0}\right|<\left|z_{2}-x_{0}\right|\right\}$ separating the circular parts of the boundary $\partial A$ in $A$. Then, by [5, p. 347, Example 1.8],

$$
m(\Gamma) \geq m\left(\Gamma_{0}\right)=\frac{1}{\pi} \log \left|\frac{z_{2}-x_{0}}{z_{1}-x_{0}}\right|
$$

which implies the left hand side of (2.5).

Next, consider the metric

$$
\rho(z):= \begin{cases}\left(\pi\left|z-x_{0}\right|\right)^{-1} & \text { if } z \in \mathbb{H}, e^{-\pi}\left|z_{1}-x_{0}\right| \leq\left|z-x_{0}\right| \leq e^{\pi}\left|z_{2}-x_{0}\right|, \\ 0 & \text { elsewhere in } \mathbb{C} .\end{cases}
$$

The analysis analogous to the proof of $[5$, p. 349, (1.11)] yields

$$
\int_{\gamma} \rho(z)|d z| \geq 1, \quad \gamma \in \Gamma
$$

i.e., $\rho$ is admissible (in the L-definition) of $m(\Gamma)$. Therefore,

$$
m(\Gamma) \leq \int \rho(z)^{2} d m(z)=\frac{1}{\pi} \log \left|\frac{z_{2}-x_{0}}{z_{1}-x_{0}}\right|+2,
$$

where $d m$ means integration with respect to the two-dimensional Lebesgue measure (area), which implies the right-hand side inequality in (2.5).

In a similar way, for $\eta_{0}:=F\left(x_{0}\right)$ and $w_{1}, w_{2} \in G$, we introduce the family $\Gamma\left(\eta_{0}, w_{1} ; w_{2}, \infty ; G\right)$ of all crosscuts of $G$ that separate points $\eta_{0}$ and $w_{1}$ from $w_{2}$ and $\infty$ in $G$.

For $r>0$, denote by $\gamma(r)=\gamma\left(\eta_{0}, G, r\right) \subset\left\{w \in G:\left|w-\eta_{0}\right|=r\right\}$ the crosscut of $G$ which has nonempty intersection with the ray $\left\{w \in \mathbb{H}: \Re(w)=\eta_{0}\right\}$. For $0<$ $r<R$, denote by $Q(r, R)=Q\left(\eta_{0}, G, r, R\right) \subset G$ the bounded simply connected domain whose boundary consists of $\gamma(r), \gamma(R)$, and two connected parts of $\partial G$. Let $m(r, R)=m\left(\eta_{0}, G, r, R\right)$ be the module of the family $\Gamma(r, R)=\Gamma\left(\eta_{0}, G, r, R\right)$ of all crosscuts of $Q(r, R)$ which separate circular arcs $\gamma(r)$ and $\gamma(R)$ in $Q(r, R)$. 
Lemma 1 For $0<r<R \leq R_{0}:=\left|w_{0}-\eta_{0}\right|$,

$$
m(r, R) \leq \frac{1}{\pi} \log \frac{R}{r}+c_{2}, \quad c_{2}:=\frac{1}{\pi} \log \frac{c^{2}}{c_{1}}+7 .
$$

Proof. From the right-hand side of $(2.5)$ we conclude that for $\Gamma_{1}:=\Gamma\left(x_{0}, z ; z_{0}, \infty ; \mathbb{H}\right)$ and $z \in \mathbb{H}$ with $\left|z-x_{0}\right|<\left|z_{0}-x_{0}\right|$,

$$
m\left(\Gamma_{1}\right) \leq \frac{1}{\pi} \log \left|\frac{z_{0}-x_{0}}{z-x_{0}}\right|+2 .
$$

While, for $\Gamma_{1}^{\prime}:=F\left(\Gamma_{1}\right)=\Gamma\left(\eta_{0}, w ; w_{0}, \infty ; G\right)$ and $w:=F(z)$, where $\left|w-\eta_{0}\right|$ is sufficiently small, we have

$$
\begin{aligned}
m\left(\Gamma_{1}^{\prime}\right) \geq & m\left(\left|w-\eta_{0}\right|, R_{0}\right) \geq m\left(\left|w-\eta_{0}\right|, r\right)+m(r, R)+m\left(R, R_{0}\right) \\
\geq & m\left(\left\{\left\{\xi \in \mathbb{H}:\left|\xi-x_{0}\right|=t\right\}:\left|w-\eta_{0}\right|<t<r\right\}\right)+m(r, R) \\
& +m\left(\left\{\left\{\xi \in \mathbb{H}:\left|\xi-x_{0}\right|=t\right\}: R<t<R_{0}\right\}\right) \\
= & \frac{1}{\pi} \log \frac{r}{\left|w-\eta_{0}\right|}+m(r, R)+\frac{1}{\pi} \log \frac{R_{0}}{R} .
\end{aligned}
$$

Since $m\left(\Gamma_{1}\right)=m\left(\Gamma_{1}^{\prime}\right)$, comparing above inequalities, we obtain

$$
\begin{aligned}
m(r, R) & \leq \frac{1}{\pi} \log \left|\frac{z_{0}-x_{0}}{z-x_{0}}\right|+2-\frac{1}{\pi} \log \frac{r}{\left|w-\eta_{0}\right|}-\frac{1}{\pi} \log \frac{R_{0}}{R} \\
& =\frac{1}{\pi} \log \left|\frac{w-\eta_{0}}{z-x_{0}}\right|+\frac{1}{\pi} \log \frac{\left|z_{0}-x_{0}\right|}{R_{0}}+2+\frac{1}{\pi} \log \frac{R}{r} .
\end{aligned}
$$

Taking limit as $z \rightarrow x_{0}$ we have

$$
m(r, R) \leq \frac{1}{\pi} \log \left|F^{\prime}\left(x_{0}\right)\right|+\frac{1}{\pi} \log \frac{\left|z_{0}-x_{0}\right|}{R_{0}}+2+\frac{1}{\pi} \log \frac{R}{r}
$$

which, together with (2.2) and (2.3), yields (2.6).

Lemma 2 For $j=1, \ldots, m-1$, we have

$$
\frac{v_{j}}{\left|\eta_{0}-u_{j}\right|}<\frac{\sqrt{\left(\eta_{0}-u_{j}\right)^{2}+v_{j}^{2}}}{\left|\eta_{0}-u_{j}\right|} \leq c_{3}:=4 \exp \left(3 \pi c_{2}\right) .
$$

Proof. Let $r_{j}:=\left|\eta_{0}-u_{j}\right|, R_{j}:=\sqrt{\left|\eta_{0}-u_{j}\right|^{2}+v_{j}^{2}}$. By Lemma 1

$$
m\left(r_{j}, R_{j}\right) \leq \frac{1}{\pi} \log \frac{R_{j}}{r_{j}}+c_{2} .
$$


Furthermore, we claim that

$$
m\left(r_{j}, R_{j}\right) \geq \frac{4}{3 \pi} \log \frac{R_{j}}{\sqrt{2} r_{j}} .
$$

Indeed, according to the nonnegativity of the module of a family of curves, there is no loss of generality in assuming that $R_{j}>\sqrt{2} r_{j}$. Since

$$
|\gamma(r)| \leq \frac{3}{4} \pi r, \quad \sqrt{2} r_{j}<r<R_{j}
$$

we have

$$
\begin{aligned}
m\left(r_{j}, R_{j}\right) & \geq m\left(\sqrt{2} r_{j}, R_{j}\right) \geq m\left(\left\{\gamma(r): \sqrt{2} r_{j}<r<R_{j}\right\}\right) \\
& \geq \int_{\sqrt{2} r_{j}}^{R_{j}} \frac{d r}{|\gamma(r)|} \geq \frac{4}{3} \int_{\sqrt{2} r_{j}}^{R_{j}} \frac{d r}{\pi r}=\frac{4}{3 \pi} \log \frac{R_{j}}{\sqrt{2} r_{j}} .
\end{aligned}
$$

Making use of (2.8) and (2.9), we obtain

$$
\frac{R_{j}}{r_{j}} \leq 4 \exp \left(3 \pi c_{2}\right)
$$

which implies (2.7).

Lemma 3 For $z \in \mathbb{H}$ with $\left|z-x_{0}\right|<2 c$,

$$
\left|F(z)-\eta_{0}\right| \leq c_{4}\left|z-x_{0}\right|, \quad c_{4}:=\frac{c_{1}+1}{c} \exp \left(\pi\left(c_{2}+4+2 \pi c_{3}^{2}\right)\right) .
$$

Proof. First, we claim that for $w:=F(z)$,

$$
\left|w-\eta_{0}\right| \leq\left|w_{0}-\eta_{0}\right| .
$$

To prove (2.11), we assume that $\left|w-\eta_{0}\right|>\left|w_{0}-\eta_{0}\right|$. Then, for the module of $\Gamma_{2}^{\prime}:=\Gamma\left(\eta_{0}, w ; w_{0}, \infty ; G\right)$ we have

$$
m\left(\Gamma_{2}^{\prime}\right) \leq 4 .
$$

Indeed, since for $\gamma \in \Gamma_{2}^{\prime}$,

$$
\gamma \cap\left\{\xi \in G:\left|\xi-\eta_{0}\right|=\mid w_{0}-\eta_{0}\right\} \neq \emptyset,
$$

the metric

$$
\rho_{1}(\xi):= \begin{cases}\pi^{-1} & \text { if } 0 \leq \Re(\xi) \leq \pi,\left|\Im\left(\xi-w_{0}\right)\right| \leq 2 \pi \\ 0 & \text { elsewhere in } \mathbb{C}\end{cases}
$$


is admissible for $\Gamma_{2}^{\prime}$. Therefore,

$$
m\left(\Gamma_{2}^{\prime}\right) \leq \int \rho_{1}(\xi)^{2} d m(\xi)=4
$$

This proves (2.12).

Moreover, according to our assumption $\left|z-x_{0}\right|<e^{-4 \pi}\left|z_{0}-x_{0}\right|$ and the left-hand side of (2.5), for the module of $\Gamma_{2}:=F^{-1}\left(\Gamma_{2}^{\prime}\right)=\Gamma\left(x_{0}, z ; z_{0}, \infty ; \mathbb{H}\right)$ we obtain

$$
m\left(\Gamma_{2}\right) \geq \frac{1}{\pi} \log \left|\frac{z_{0}-x_{0}}{z-x_{0}}\right|>4,
$$

which contradicts (2.12). Hence, (2.11) holds.

Our next objective is to estimate from above the module of $\Gamma_{2}^{\prime}$ under the assumption (2.11). Denote by $r(w)$ the supremum of values of $r>0$ such that $\gamma(r)$ separates $\eta_{0}$ and $w$ in $G$. By Lemma 2

$$
\left|w-\eta_{0}\right| \leq r(w) \leq c_{3}\left|w-\eta_{0}\right|
$$

Let $\rho_{2}$ be the extremal metric for the family $\Gamma_{3}:=\Gamma\left(r(w),\left|\eta_{0}-w_{0}\right|\right)$, i.e.,

$$
\begin{gathered}
\int_{\gamma} \rho_{2}(\xi)|d \xi| \geq 1, \quad \gamma \in \Gamma_{3}, \\
m\left(r(w),\left|\eta_{0}-w_{0}\right|\right)=\int \rho_{2}(\xi)^{2} d m(\xi) .
\end{gathered}
$$

Since for $\gamma \in \Gamma_{2}^{\prime}$ with $\gamma \cap \gamma(r(w)) \neq \emptyset$ we have $|\gamma| \geq r(w) / c_{3}$, for such $\gamma$ and the metric

$$
\rho_{3}(\xi):= \begin{cases}\frac{c_{3}}{r(w)} & \text { if } \xi \in G,\left|\xi-\eta_{0}\right| \leq 2 r(w), \\ 0 & \text { elsewhere in } \mathbb{C}\end{cases}
$$

we obtain

$$
\int_{\gamma} \rho_{3}(\xi)|d \xi| \geq 1
$$

From what we already proved, we conclude that the metric

$$
\rho(\xi):=\max _{k=1,2,3} \rho_{k}(\xi), \quad \xi \in \mathbb{C}
$$

is admissible for $\Gamma_{2}^{\prime}$ and, by (2.6) and (2.14),

$$
\begin{aligned}
m\left(\Gamma_{2}^{\prime}\right) & \leq \sum_{k=1}^{3} \int \rho_{k}(\xi)^{2} d m(\xi) \leq 4+\frac{1}{\pi} \log \frac{\left|w_{0}-\eta_{0}\right|}{r(w)}+c_{2}+2 \pi c_{3}^{2} \\
& \leq \frac{1}{\pi} \log \frac{\left|w_{0}-\eta_{0}\right|}{\left|w-\eta_{0}\right|}+c_{5}, \quad c_{5}:=c_{2}+4+2 \pi c_{3}^{2} .
\end{aligned}
$$


Comparing the above inequality with (2.13) and using (2.4), we obtain

$$
\left|w-\eta_{0}\right| \leq \frac{\left|w_{0}-\eta_{0}\right|}{\left|z_{0}-x_{0}\right|} e^{c_{5} \pi}\left|z-x_{0}\right|<\frac{c_{1}+1}{c} e^{c_{5} \pi}\left|z-x_{0}\right|,
$$

which implies (2.10).

\section{Proof of Theorem 1}

Neither the hypothesis nor the conclusion is affected if we assume that $E \subset$ $[-1,1], \pm 1 \in E$ and $-1<x_{0}<1$. Next, we construct a sequence $E_{j}, j \in \mathbb{N}$ of compact sets each of which consists of a finite number of intervals such that $x_{0} \in$ $\operatorname{Int}\left(E_{j}\right)$ for each $j$ and

$$
E_{j+1} \subset E_{j} \subset[-1,1], \quad \bigcap_{j=1}^{\infty} E_{j}=E .
$$

By [12, p. 108, Theorem 4.4.6]

$$
\lim _{j \rightarrow \infty} g_{\overline{\mathbb{C}} \backslash E_{j}}(z)=g_{\overline{\mathbb{C}} \backslash E}(z), \quad z \in \mathbb{C} \backslash E .
$$

Since $\mathcal{E}_{n}\left(\left|x-x_{0}\right|^{\alpha}, E\right) \leq \mathcal{E}_{n}\left(\left|x-x_{0}\right|^{\alpha}, E_{j}\right)$, by assumption (1.1) and the VasilievTotik theorem (2.1), it follows that for some $\alpha>0$,

$$
h_{E_{j}}\left(x_{0}\right)^{-\alpha} \sigma_{\alpha}=\lim _{n \rightarrow \infty} n^{\alpha} \mathcal{E}_{n}\left(\left|x-x_{0}\right|^{\alpha}, E_{j}\right) \geq \limsup _{n \rightarrow \infty} n^{\alpha} \mathcal{E}_{n}\left(\left|x-x_{0}\right|^{\alpha}, E\right)=: \beta>0 .
$$

Hence,

$$
h_{E_{j}}\left(x_{0}\right) \leq\left(\frac{\sigma_{\alpha}}{\beta}\right)^{1 / \alpha}, \quad \operatorname{cap}\left(E_{j}\right) \geq \operatorname{cap}(E),
$$

and (2.2) holds for each $E_{j}$ instead of $E$ with a constant $c>2$ independent of $j$. Referring to Lemma 3, we find that for $z \in \mathbb{C} \backslash E_{j}$ with $\left|z-x_{0}\right| \leq 2 c$,

$$
g_{\overline{\mathbb{C}} \backslash E_{j}}(z) \leq c_{4}\left|z-x_{0}\right|
$$

where a constant $c_{4}$ also does not depend on $j$. A passage to the limit as $j \rightarrow \infty$ and (3.1) yield

$$
g_{\overline{\mathbb{C}} \backslash E}(z) \leq c_{4}\left|z-x_{0}\right|
$$

Next, consider the case where $\left|z-x_{0}\right| \geq 2 c>4$. By [13, p. 53], in this case

$$
g_{\overline{\mathbb{C}} \backslash E}(z)=\int \log |z-x| d \mu_{E}(x)-\log \operatorname{cap}(E) \leq \log \left(2 c\left|z-x_{0}\right|\right)
$$

which, together with (3.2), implies (1.2) 
This concludes the proof of Theorem 1 .

\section{Acknowledgements}

The author is grateful to M. Nesterenko for his helpful comments.

\section{References}

[1] Ahlfors L. V. (1973)

Conformal invariants, McGraw-Hill, New York.

[2] Andrievskii V. V. (2001)

A Remez-type inequality in terms of capacity, Complex Variables 45, 35-46.

[3] Andrievskii V. V. (2008)

Positive harmonic functions on Denjoy domains in the complex plane, J. d'Analyse Math. 104, 83-124.

[4] Andrievskii V. V. (2009)

Polynomial approximation of piecewise analytic functions on a compact subset of the real line, J. Approx. Theory 161, 634-644.

[5] Andrievskii V. V. and H. -P. Blatt (2002)

Discrepancy of signed measures and polynomial approximation, SpringerVerlag, Berlin/New York.

[6] Bernstein S. (1914)

Sur la meilleure approximation de $|x|$ par des polynomes des degrés donnés, Acta Math. 37, 1-57.

[7] Bernstein S. (1938)

On the best approximation of $|x|^{p}$ by means of polynomials of extremely high degree, Izv. Akad. Nauk SSSR Ser. Mat. 2, 160-180 (in Russian).

[8] Bernstein S. (1938)

On the best approximation of $|x-c|^{p}$, Dokl. Akad. Nauk SSSR 18, 379-384 (in Russian). 
[9] Carleson L. and V. Totik (2004)

Hölder continuity of Green's functions, Acta Sci. Math. (Szeged) 70, 557608.

[10] Carroll T. and S. Gardiner (2008)

Lipschitz continuity of the Green function in Denjoy domains, Ark. Mat. 46, 271-283.

[11] Pommerenke Ch. (1992)

Boundary behavior of conformal maps, Vandenhoeck and Ruprecht, Göttingen.

[12] Ransford T. (1995)

Potential theory in the complex plane, Cambridge University Press, Cambridge.

[13] Saff E. B. and V. Totik (1997)

Logarithmic potentials with external fields, Springer-Verlag, New York/Berlin.

[14] Totik V. (2006)

Metric properties of harmonic measures, Mem. Amer. Math. Soc. 184(867).

[15] Vasiliev R. K. (1998)

Chebyshev polynomials and approximation theory on compact subsets of the real axis, Saratov University Publishing House, Saratov.

[16] Widom H. (1969)

Extremal polynomials associated with a system of curves in the complex plane, Adv. in Math. 3, 127-232.

V. V. Andrievskii

Department of Mathematical Sciences

Kent State University

Kent, OH 44242

USA

e-mail: andriyev@math.kent.edu 